\title{
ON THE REPRESENTATION OF ANALYTIC FUNCTIONS OF SEVERAL VARIABLES AS INFINITE PRODUCTS*
}

\author{
BY J. M. FELD AND P. NEWMAN
}

1. Introduction. In a paper by J. F. Ritt soon to appear in the Mathematische Zeitschrift, he proves that any function $f(z)$, analytic, and equal to unity at $z=0$, can be represented in one and only one way as an infinite product $\Pi_{1}^{\infty}\left(1+c_{n} z^{n}\right)$, which converges absolutely for $|z| \leqq 1 /(6 R)$, where $R$ is the least upper bound of the. infinite sequence $\left|b_{1}\right|,\left|b_{2}\right| 1 / 2, \cdots$, $\left|b_{k}\right|^{1 / k}, \cdots$ and $b_{k}$ is the coefficient of $z^{k-1}$ in the Taylor expansion of $f^{\prime}(z) / f(z)$.

The object of this paper is to extend this result to functions of two variables. The first part will be concerned with a demonstration that an analytic function $f(x, y)=1+\sum b_{m n} x^{m} y^{n}$ can be uniquely represented as an absolutely convergent infinite product $\Pi\left(1+a_{m n} x^{m} y^{n}\right)$ with constant $a$ 's. The second part will consider the representation of $f(x, y)$ in the form $\Pi_{1}^{\infty}\left(1+P_{n}\right)$ where $P_{n}$ is a homogeneous polynomial in $x$ and $y$ of degree $n$. Although the proof in each case is carried out for two variables, it will be evident how to extend it to functions of any number of variables. It should be noted, however, that the analytic functions considered in this paper constitute a restricted class of such functions, namely, those which equal unity at the origin. For one variable the corresponding assumption is not an essentially restrictive one.

2. THEOREM 1. If $f(x, y)$ is analytic and equal to unity at $(0,0)$, then in the neighborhood of $(0,0)$ it admits a unique representation as an absolutely convergent infinite product

with constant $a_{m n}$

$$
f(x, y)=\prod_{m, n}\left(1+a_{m n} x^{m} y^{n}\right)
$$

Let the Taylor's expansion of $f(x, y)$ at $(0,0)$ be $1+\sum b_{m n} x^{m} y^{n}$. Since $f(0,0)=1$ there is a neighborhood about $(0,0)$ for which

\footnotetext{
* Presented to the Society, October 26, 1929.
} 
$\log f(x, y)$ will be represented by an absolutely convergent expansion $\sum C_{m n} x^{m} y^{n}$. By Lemaire's generalization of a theorem of Cauchy (Bulletin des Sciences Mathématiques, 1896, p. 286), the set $\left|C_{m n}\right|^{1 /(m+n)}$ is bounded; we denote the least upper bound by $r$.

Assuming the product expansion and taking logarithms, we. find

$$
\sum_{m, n} C_{m n} x^{m} y^{n}=\sum_{m, n, p}(-1)^{p+1} \frac{\left(a_{m n} x^{m} y^{n}\right)^{p}}{p} .
$$

We denote the common divisors of a fixed pair of $m$ and $n$ by $d_{0}=1, d_{1}, \cdots, d_{t}=D$, where $D$ is the greatest common divisor. Observing that

$$
\frac{\left(a_{m / d_{i}, n / d_{i}} x^{m / d_{i}} y^{n / d_{i}}\right)^{d_{i}}}{d_{i}}=\frac{\left(a_{m / d_{i}, n / d_{i}}\right)^{d_{i}} x^{m} y^{n}}{d_{i}},
$$

we find for the coefficient of $x^{m} y^{n}$ in the second member of (1),

$$
\sum_{i=0}^{t}(-1)^{d_{i}+1} \frac{1}{d_{i}}\left(a_{m / d_{i}, n / d_{i}}\right)^{d_{i}} .
$$

Equating coefficients of $x^{m} y^{n}$, we have

$$
C_{m n}=a_{m n}+\sum_{1}^{t} \pm \frac{1}{d_{i}}\left(a_{m / d_{i}, n / d_{i}}\right)^{d_{i}} .
$$

If neither $m$ nor $n$ is zero, then (2) will not involve any $a$ with a zero subscript. This is also evident from the consideration that a zero subscript represents a term in the product of the form $\left(1+a_{0, n} y^{n}\right)$ which could not give rise to a term containing $x$ when expanded in a logarithmic series. It follows from our hypothesis that the subscripts 00 never occur.

When $m$ or $n$ is unity, (2) reduces to

$$
C_{1 n}=a_{1 n} \text {. }
$$

From (2) we get

$$
\left|a_{m n}\right| \leqq\left|C_{m n}\right|+\sum_{1}^{t} \frac{1}{d_{i}}\left|a_{m / d_{i}, n / d_{i}}\right|^{d_{i}} .
$$

If $\left(1 / d_{k}\right)\left|a_{m / d_{k}, n / d_{k}}\right| d_{k}$ is the greatest of the terms following $C_{m n}$ in (4), then since the number of common divisors cannot exceed $D / 2$, we obtain 


$$
\left|a_{m n}\right| \leqq\left|C_{m n}+\frac{D}{2}\right| a_{m / d_{k}, n / d_{k}} \mid d_{k} .
$$

There are two possibilities to be considered in (5); (A) if the second term on the right of (4) is less than or equal to the first, then $\left|a_{m n}\right|^{1 /(m+n)} \leqq\left(2\left|C_{m n}\right|\right)^{1 /(m+n)} \leqq 2 r$, otherwise, (B)

$$
\left|a_{m n}\right|<D\left|a_{m / d_{k}, n / d_{k}}\right|^{d_{k}} \text {. }
$$

Since $D^{1 /(m+n)} \leqq D^{1 / D}$, we find from (B)

$$
\left|a_{m n}\right|^{1 /(m+n)}<D^{1 / D}\left|a_{m / d_{k}, n / d_{k}}\right|^{d_{k} /(m+n)} .
$$

We can repeat the reasoning of $(A)$ and $(B)$ employing

$$
\left|a_{m / d_{k}, n / d_{k}}\right|^{d_{k} /(m+n)}=\left|a_{m / d_{k}, n / d_{k}}\right|^{1 /\left(m / d_{k}+n / d_{k}\right)} \text {. }
$$

If (B) holds again, the inequality of the type (6) will now involve the highest common divisor of $m / d_{k}, n / d_{k}$, say $D_{1}$. Since $d_{k} \neq 1, D \geqq 2 D_{1}$. Repeating the process until we come to an " $a$ " for which (A) holds, we have from (A) and (6)

$$
\left|a_{m n}\right|^{1 /(m+n)}<D^{1 / D} D_{1}^{1 / D_{1}} \cdots D_{l}^{1 / D_{l}}(2 r) .
$$

The $D$ 's are a decreasing sequence, $D_{j+1} \leqq 2 D_{j}$, which at the worst will end at $D_{l}=2$. This is seen readily from (3), (5), and condition (A).

From (7), we find

$$
\log \left|a_{m n}\right| 1 /(m+n)<\frac{1}{D} \log D+\frac{1}{D_{1}} \log D_{1}+\cdots+\log 2 r .
$$

We consider first the case $D_{l} \neq 3$, and since $D_{j+1} \leqq 2 D_{j}$, we have

$$
\begin{aligned}
\log \left|a_{m n}\right| 1 /(m+n)<\log 2 r+\sum_{1}^{\infty} \frac{1}{2^{k}} \log 2^{k} & =\log 2 r+\log 4 \\
& =\log 8 r .
\end{aligned}
$$

When $D_{l}=3$,

$$
\begin{aligned}
& \log \left|a_{m n}\right| 1 /(m+n)<\log 2 r+\sum_{0}^{\infty} \frac{1}{3 \cdot 2^{k}} \log \left(3 \cdot 2^{k}\right) \\
& =\log 2 r+\frac{1}{3}(\log 3) \sum_{0}^{\infty} \frac{1}{2^{k}}+\frac{1}{3} \sum_{1}^{\infty} \frac{\log 2^{k}}{2^{k}} \\
& =\log 2 r+\frac{2}{3} \log 3+\frac{1}{3} \log 4<\log 8 r .
\end{aligned}
$$


In any case,

$$
\left|a_{m n}\right| .1 /(m+n)<8 r .
$$

By Lemaire's theorem, the associated power series $\sum a_{m n} x^{m} y^{n}$ is absolutely convergent for the associated regions whose radii are given by $|\rho|\left|\rho^{\prime}\right|<1 /(8 r)$ and therefore in any such pair of regions $f(x, y)$ will be represented by the absolutely convergent product $\Pi\left(1+a_{m n} x^{m} y^{n}\right)$. From the form of the recursion formula it follows at once that the representation is unique. The theorem can be extended to any number of variables by a similar proof.

3. THEOREM 2. If $f(x, y)$ is analytic in the neighborhood of the origin and if $f(0,0)=1$, there exists a region $|x| \leqq \rho,|y| \leqq \rho$ in which $f(x, y)$ can be represented in one and only one way as an absolutely convergent infinite product $\Pi_{1}^{\infty}\left(1+Q_{n}\right)$, where $Q_{n}$ is a homogeneous polynomial in $x$ and $y$ of degree $n$.

Let the Taylor expansion of $f(x, y)$ at $(0,0)$ be written in the form

$$
f(x, y)=1+P_{1}+P_{2}+\cdots+P_{n}+\cdots,
$$

where $P_{n}$ is a homogeneous polynomial in $x$ and $y$ of degree $n$. Then there exists a $\rho_{1}$ such that (1) converges absolutely for $|x|<\rho_{1}$ and $|y|<\rho_{1}$. The function of $x, y$ and $t, f(x t, y t)$ is analytic for $x, y$ and $t$ small and has the expansion

$$
f(x t, y t)=1+\sum_{n=1}^{\infty} P_{n} t^{n} .
$$

For small values of $x, y$ and $t, \log f(x t, y t)$ is analytic and has an expansion

$$
\log f(x t, y t)=A_{1} t+A_{2} t^{2}+\cdots+A_{n} t^{n}+\cdots,
$$

where $A_{n}$ is a homogeneous polynomial in $x$ and $y$ of degree $n$, and where the set of functions $\left|A_{n}\right|^{1 / n}$ has an upper bound. Let $r$ be the least upper bound of this set in the region in which (9) holds.

Setting

$$
1+\sum_{1}^{\infty} P_{n} t^{n}=\prod_{1}^{\infty}\left(1+Q_{n} t^{n}\right)
$$

we obtain $P_{1}=Q_{1}, P_{2}=Q_{2}, P_{3}=Q_{3}+Q_{1} Q_{2}$, etc., and it is evident 
that we can solve for all the $Q$ 's by means of recursion formulas, and that $Q_{n}$ is homogeneous in $x$ and $y$ of degree $n$. Taking logarithms of both members of (10) and equating like powers of $t$, we get

$$
A_{n}=Q_{n} \pm \frac{1}{d_{1}} Q_{n / d_{1}}^{d_{1}} \pm \frac{1}{d_{2}} Q_{n / d_{2}}^{d_{2}} \pm \cdots \pm \frac{1}{n} Q^{n}
$$

where $d_{i}$ is an integral divisor of $n$. Therefore

where

$$
\left|Q_{n}\right| \leqq\left|A_{n}\right|+\frac{n}{2}\left|\frac{1}{D_{1}} Q_{n / D_{1}}^{D_{1}}\right|
$$

$$
\left|\frac{1}{D_{1}} Q_{n / D_{1}}^{D_{1}}\right|
$$

is the greatest of the terms

$$
\left|\frac{1}{d_{i}} Q_{n / d_{i}}^{d_{i}}\right|, \quad\left(d_{i}>1\right) .
$$

By a procedure like that used in the proof of Theorem 1, we arrive at the inequality

$$
\left|Q_{n}\right|^{1 / n}<n^{1 / n} D^{1 / D_{1}} D^{1 / D_{2}} \cdots D^{1 / D_{i}}(2 r) .
$$

We therefore have, as in the preceding case, $\left|Q_{n}\right|^{1 / n}<8 r$. Thus there exists a $\rho_{2}$ such that $\sum\left|Q_{n}\right| t^{n}$ converges for $|x| \leqq \rho_{2}$, $|y| \leqq \rho_{2},|t| \leqq 1 /(8 r)$. Therefore $\Pi\left(1+Q_{n} t^{n}\right)$ must converge absolutely in this region. Since

$$
f(x t, y t)=\Pi\left[1+Q_{m}(x t, y t)\right],
$$

we have the result that

$$
f(x, y)=\Pi\left(1+Q_{n}\right)
$$

in the region $|x| \leqq \rho=\rho_{2} /(8 r),|y| \leqq \rho=\rho_{2} /(8 r)$.

Columbia University 